\title{
Vegetative Compatibility Groups and Aggressiveness of North American Isolates of Colletotrichum coccodes, the Causal Agent of Potato Black Dot
}

\author{
N. Nitzan, Department of Plant Pathology, Washington State University, Pullman 99164-6430; L. Tsror (Lahkim), \\ Department of Plant Pathology, Agriculture Research Organization, Gilat Experiment Station, M. P. Negev, 85280, \\ Israel; and D. A. Johnson, Department of Plant Pathology, Washington State University, Pullman
}

\begin{abstract}
Nitzan, N., Tsror (Lahkim), L., and Johnson, D. A. 2006. Vegetative compatibility groups and aggressiveness of North American isolates of Colletotrichum coccodes, the causal agent of potato black dot. Plant Dis. 90:1287-1292.

The vegetative compatibility of 123 isolates of Colletotrichum coccodes from North America (United States and Canada) originating from potato, tomato, pepper, and mint was tested using nitrate-nonutilizing (nit) mutants. The North American isolates did not anastomose with previously selected European/Israeli vegetative compatibility group (VCG) testers; therefore, eight isolates were selected as VCG testers for the North American population. The 123 isolates distributed to seven VCGs at 1.6, 1.6, 4.0, 8.1, 13.8, 19.5, and 36.6\%, with $14.6 \%$ of the isolates not assigned to any of the seven VCGs. Among the North American (NA)-VCGs, the average frequency of the nit1/nit3 nit mutants was lower $(P<0.05)$ for isolates belonging to NA-VCG1 than for isolates belonging to the NA-VCGs 2, 3, and 5. In contrast, the frequency of NitM nit mutants did not vary $(P>0.05)$ among the NA-VCGs and was collectively $5.14 \%$. The results also indicated significant $(P<0.05)$ differences among NA-VCGs and European/Israeli (EU/I)VCGs regarding the frequency of nit mutants. The aggressiveness trials of the North American isolates to potato indicated that plants infected with isolates belonging to NA-VCG2 and NAVCG5 had more $(P<0.05)$ sclerotia on the roots and crowns than plants infected with isolates belonging to NA-VCGs 1 and 3. The plants infected with isolates belonging to NA-VCG2 had sclerotia formed higher $(P<0.05)$ up the stem than the plants infected with isolates belonging to NA-VCGs 1,3 , or 5. The plants infected with isolates assigned to NA-VCG2 had more $(P<$ 0.05 ) infected progeny tubers than the plants infected with isolates belonging to NA-VCGs 1,3 , or 5; and the plants infected with isolates belonging to NA-VCGs 1,2 , and 5 yielded fewer $(P<$ 0.05 ) potato tubers than the noninoculated control plants. A naming system for the population of C. coccodes based on the continent source of the population, the VCG number, and the isolate's code was suggested.
\end{abstract}

Additional keyword: pathogenicity

Potato black dot is caused by the tuber-, soil-, and airborne pathogen Colletotrichum coccodes (Wallr.) S. J. Hughes (syn. C. atramentarium (Berk. \& Broome) Taubenhaus). The disease is characterized by small black sclerotia developing on roots, stems, stolons, and the surface of progeny potato tubers of senescing and dead potato plants $(1,4,7,19)$. Black dot is common in most potato-growing areas in the world $(1,2,5,6,12,14)$ and can cause up to $30 \%$ yield reduction and reduce tuber quality on susceptible cultivars $(9,15,18-$ 20).

C. coccodes lacks a recognized teleomorph; therefore, exchange of genetic

\section{Corresponding author: N. Nitzan}

E-mail: nun2@psu.edu

The following research was assigned Plant Pathology New Series No. 0389.

Accepted for publication 1 May 2006.

DOI: 10.1094/PD-90-1287

(C) 2006 The American Phytopathological Society material among isolates probably is restricted to the vegetative compatibility system. Vegetative compatibility is a genetically determined ability in which isolates of the same fungal species anastomose and form stable heterokaryons, during which genetic material may transfer from one isolate to another (13). Isolates which are vegetatively compatible may form subspecific populations that tend to be genetically isolated in nature and are called vegetative compatibility groups (VCGs; 13). Isolates belonging to a VCG tend to have a common genetic pool (13) and, therefore, may have similar pathological and physiological traits differing from those of isolates that do not belong to that VCG $(8,13,16,17)$.

Testing isolates for VCGs usually is based on complementation tests with nitrate-nonutilizing (nit) mutants $(8,11,14)$, which are impaired in their ability to utilize nitrate from the growing media. When the hyphae of two different nit mutants anastomose to form stable heterokaryons, a biochemical complementation takes place in the heterokaryons which allows them to utilize nitrate when it is the sole nitrogen source in the growing media. The result of the complementation is visible to the naked eye as a prototrophic growth at the contact zone of the mutants' colonies. However, the formation of heterokaryons and, consequentially, the biochemical complementation and prototrophic growth are contingent upon the vegetative compatibility of the isolates from which the mutants were derived. If the isolates were vegetatively incompatible, stable heterokaryons would not have been formed and a prototrophic growth would not have been evident. Hence, testing isolates for vegetative compatibility using nit mutants is a technique based upon a biological response which genetically is restricted, and its results tend to be clear-cut (13).

In a previous study (14), we collected 110 isolates of $C$. coccodes from European and Israeli potato tubers, roots, and stems and tested their vegetative compatibility. In all, 36 isolates were from Israel, 23 from France, and 51 from the Netherlands. The study resulted in the characterization of four multimember VCGs among the 110 isolates, and the selection of 12 different isolates as VCG testers for the European/Israeli population (14). The European/Israeli (EU/I)-VCG1 consisted of eight isolates, in which two were from Israel, two from the Netherlands, and four from France. EU/I-VCG2 was the largest VCG consisting, of 39 isolates, of which 7 were from Israel, 17 from the Netherlands, and 15 from France. EU/I-VCG3 was the second largest, consisting of 22 isolates, of which 11 were from Israel and 11 from the Netherlands, and EU/I-VCG 4 consisted of 11 isolates, of which 2 were from Israel and 9 from the Netherlands (14). The potential aggressiveness of the isolates from the different EU/I-VCGs to potato was evaluated using the susceptible potato cvs. Desiree and Mondial. Potato plants were inoculated with 34 isolates that were selected from the different European/Israeli VCGs. The results indicated that, on average, the isolates belonging to EU/I-VCG 3 were more aggressive than the isolates of the other EU/I-VCGs (14). Furthermore, in vitro growth rate trials which tested the response of the different isolates to temperature $\left(21,25\right.$, and $\left.30^{\circ} \mathrm{C}\right)$ and $\mathrm{pH}(\mathrm{pH} 5$, 6 , and 7) indicated that a physiological variation among the VCGs also was present (15). The results indicated that the 
subspecific level of $C$. coccodes may significantly affect disease epidemiology and etiology and, consequentially, disease management; and strengthened the hypothesis that differences in aggressiveness could be correlated with differences in vegetative compatibility or with the subspecific level of the pathogen.

Isolates from different VCGs may possess different epidemiological traits; therefore, a thorough understanding of the diversity in the population of $C$. coccodes would have an important and practical contribution to the improvement of disease management. More accurate procedures could be developed to screen for black dot resistance in potato lines, to screen for resistance to fungicides in the pathogen's population, and to more accurately forecast disease potential. The present study tested the hypothesis that the population of $C$. coccodes in North America was composed of several distinct VCGs with diverse genetic and pathological traits. The main objective of the study was to characterize VCGs and pathogenicity-correlated traits in the North American population of $C$. coccodes, in order to provide a basis for a thorough comparison between the European/Israeli and North American populations.

\section{MATERIALS AND METHODS}

Collection of North American C. coccodes isolates. In all, 123 isolates that were collected from 13 states in the United States and 3 provinces in Canada between 1986 and 2002 were studied. Of these, 115 isolates originated from potato plants and tubers, 3 from tomato plants and fruit, 4 from peppermint, and 1 from red pepper (Table 1). All isolates, including those obtained from other laboratories, were subcultured to $1 \%$ potato dextrose agar
(PDA, 10 g/liter, Bacto agar, 15 g/liter, and streptomycin sulfate, $0.1 \mathrm{~g} /$ liter) and were incubated in the dark at $25^{\circ} \mathrm{C}$ for 7 days. Monoconidial cultures were obtained for each isolate by streaking a mass of conidia onto Czapek Dox agar (CDA) in 9-cm petri plates and incubating at $25^{\circ} \mathrm{C}$ in the dark for $24 \mathrm{~h}$. A single conidium was transferred under the microscope onto CDA in $5-\mathrm{cm}$ petri plate and incubated at $25^{\circ} \mathrm{C}$ in the dark for 7 days. The monoconidial cultures were maintained at $10^{\circ} \mathrm{C}$. All of the experiments were performed using the monoconidial cultures, which are referred to as isolates.

nit Mutants. The nit mutants were generated to all isolates by transferring agar blocks $\left(1 \mathrm{~cm}^{2}\right)$ that were colonized by the fungus to water agar chlorate (WAC) medium (2\% Bacto agar, $3 \%$ potassium chlorate, and $0.02 \%$ glucose; 10 ) in $9-\mathrm{cm}$ plates and were incubate in the dark at $25^{\circ} \mathrm{C}$ for 21 days (14). In all, 10 to 20 agar blocks (replications) were transferred to WAC for each isolate. After 21 days of incubation, hyphae from every replication were transferred to CDA in 9-cm plates by streaking, and the plates were reincubated for 7 days in the dark at $25^{\circ} \mathrm{C}$. Colonies that had a thin, transparent white or green mycelium were considered nit mutants. A single spore colony from each suspected nit mutant was retransferred to CDA in 5$\mathrm{cm}$ plates and incubated for 7 days in the dark at $25^{\circ} \mathrm{C}$ to assure the mutant's stability. Partial characterization of the nit phenotype (nit1/nit3 or NitM) was followed by placing two mycelia blocks of nit mutant on both CDA and CDA amended with $0.02 \%$ hypoxanthine, and incubating in the dark for 5 days at $25^{\circ} \mathrm{C}$. Colonies with a prototrophic growth on CDA supplemented with hypoxanthine and with a thin mycelium on CDA were characterized as nit1/nit3 mutants, and colonies with thin mycelia on both media were characterized as NitM mutants $(3,11,14)$.

Complementation tests. The EU/I VCG tester isolates, previously selected by Nitzan et al. (14), were used to characterize the North American (NA) isolates to VCGs. The EU/I testers were isolates 2 and 16 from EU/I-VCG 1; isolates 3, 12, $13,18,24$, and 53 from EU/I-VCG 2; isolates 38 and 138 from EU/I-VCG 3; and isolates 46 and 49 from EU/I-VCG 4. Complementation tests between nit mutants were performed on CDA, which had only nitrate as the nitrogen source. Agar blocks $\left(5 \mathrm{~mm}^{2}\right)$ colonized with mycelia of the NitM and nitl/nit3 mutants of the tested isolates were placed $1 \mathrm{~cm}$ apart in 5$\mathrm{cm}$ petri plates. The plates were incubated for 14 days at $25^{\circ} \mathrm{C}$ in the dark. Complementation was characterized by the formation of a prototrophic growth at the contact zone of the colonies of the two mutants, and was evident after 10 to 14 days.

Aggressiveness trials. The trials were conducted in a greenhouse on the Pullman campus of Washington State University during 2003 and were repeated in 2004 with the same isolates. The temperatures during the trials were $18 \pm 4.4$ and $18 \pm$ $4.9^{\circ} \mathrm{C}$ in 2003 and 2004 , respectively. The photoperiod during the trials was $16 \mathrm{~h}$ of light and $8 \mathrm{~h}$ of dark. The trials were conducted in 11-liter pots that were filled with Sunshine LC1 potting mixture (Sun-Gro Horticulture Distributor Inc., Bellevue, WA). The experimental design structure was a randomized complete block design with seven replications per isolate, including noninoculated control plants.

Tested isolates. In all, 41 isolates (34\% of the collection) arbitrarily selected from the NA-VCGs were tested for aggressiveness to potato. Of these, 36 isolates origi-

Table 1. Distribution of Colletotrichum coccodes isolates from different locations in North America to vegetative compatibility groups (VCGs)

\begin{tabular}{|c|c|c|c|c|c|c|c|c|c|}
\hline \multirow[b]{2}{*}{ Location } & \multicolumn{7}{|c|}{ North American (NA)-VCGs } & \multirow[b]{2}{*}{ Nonassigned } & \multirow[b]{2}{*}{ Total tested $^{\mathrm{z}}$} \\
\hline & 1 & 2 & 3 & 4 & 5 & 6 & 7 & & \\
\hline Idaho & 1 & 5 & 1 & 0 & 3 & 0 & 0 & 2 & 12 \\
\hline Minnesota & 0 & 1 & 1 & 0 & 0 & 0 & 0 & 0 & 2 \\
\hline Montana & 6 & 6 & 1 & 0 & 6 & 0 & 0 & 6 & 25 \\
\hline Nebraska & 1 & 0 & 0 & 1 & 0 & 0 & 0 & 0 & 2 \\
\hline Nevada & 1 & 1 & 2 & 0 & 1 & 0 & 0 & 1 & 6 \\
\hline New York & 0 & 1 & 0 & 0 & 0 & 0 & 2 & 0 & 3 \\
\hline North Dakota & 7 & 3 & 1 & 0 & 0 & 0 & 0 & 0 & 11 \\
\hline Ohio & 0 & 0 & 0 & 0 & 0 & 2 & 0 & 0 & 2 \\
\hline Oregon & 0 & 4 & 0 & 1 & 2 & 0 & 0 & 3 & 10 \\
\hline Pennsylvania & 0 & 1 & 0 & 0 & 0 & 0 & 0 & 1 & 2 \\
\hline Texas & 0 & 0 & 0 & 0 & 1 & 0 & 0 & 0 & 1 \\
\hline Washington & 7 & 15 & 2 & 0 & 2 & 0 & 2 & 3 & 31 \\
\hline Wisconsin & 0 & 0 & 0 & 0 & 2 & 0 & 1 & 1 & 4 \\
\hline Alberta & 0 & 6 & 1 & 0 & 0 & 0 & 0 & 1 & 8 \\
\hline British Columbia & 0 & 0 & 1 & 0 & 0 & 0 & 0 & 0 & 1 \\
\hline Saskatchewan & 1 & 0 & 0 & 0 & 0 & 0 & 0 & 0 & 1 \\
\hline Not recorded & 0 & 2 & 0 & 0 & 0 & 0 & 0 & 0 & 2 \\
\hline Total & 24 & 45 & 10 & 2 & 17 & 2 & 5 & 18 & 123 \\
\hline
\end{tabular}

y All isolates were recovered from potato except (i) in NA-VCG2, 1 of 15 isolates from Washington was from mint; (ii) In NA-VCG6, isolates originated from tomato and red pepper; (iii) in NA-VCG7, 2 isolates from Washington and 1 from Wisconsin were from mint and 2 isolates from New York were from tomato; and (iv) among the nonassigned isolates, 1 isolate from Wisconsin was from mint.

z Total number of isolates tested. 
nated from potato, 2 from tomato, 1 from red-pepper, and 1 from mint. Of 24 isolates, 9 were tested from NA-VCG1, 16 of 45 isolates were tested from NA-VCG2, 4 of 10 isolates were tested from NA-VCG3, 2 of 2 isolates were tested from NAVCG4, 4 of 17 isolates were tested from NA-VCG5, 2 of 2 isolates were tested from NA-VCG6, and 2 of 5 isolates were tested from NA-VCG7. In addition, 1 isolate that was nonassigned to an NA-VCG and originated from potato also was tested.

Plant material. Disease-free, certified nuclear potato tubers of the $C$. coccodessusceptible cv. Russet Norkotah were used to test the aggressiveness of the selected isolates. These certified nuclear tubers were produced in a greenhouse in Montana by a commercial potato seed-tuber grower. Certified nuclear tubers were the progeny of certified tissue-cultured potato plantlets and were free of fungal, bacterial, or viral potato diseases. Both years, prior to planting, we tested a sample of 20 nuclear tubers for the presence of $C$. coccodes and other fungal pathogens of potato. The results indicated that the nuclear tubers were free of fungal and bacterial potato diseases.

Preparation of inoculum. Sterile organic rye seed were used to carry $C$. coccodes inoculum in the soil. The rye seed were washed thoroughly with distilled water to remove dirt, then transferred into 1-liter flasks. The flasks were filled to only one-third of their volume to prevent anaerobic conditions. The flasks were caped with cotton wool and aluminum foil and autoclaved for $45 \mathrm{~min}$ at $120^{\circ} \mathrm{C}$. Following sterilization, the flasks were incubated for $24 \mathrm{~h}$ in the dark at $25^{\circ} \mathrm{C}$ and reautoclaved for $45 \mathrm{~min}$ at $120^{\circ} \mathrm{C}$. The 41 isolates that were selected for the aggressiveness trials were subcultured on $1 \%$ PDA in 9-cm petri plates for 7 days at $25^{\circ} \mathrm{C}$ in the dark. The agar media of one petri plate that was fully colonized with the fungus was cut into 1$\mathrm{cm}^{2}$ blocks and transferred into a flask under sterile conditions. The flasks were recapped with autoclaved cotton wool and aluminum foil, shaken well, and incubated at $25^{\circ} \mathrm{C}$ in the dark for 3 weeks. White mycelia and black sclerotia were observed on the colonized rye seed 3 weeks after inoculation. Following the incubation period, the rye seed were dried at room temperature for 14 days in plastic boxes that were kept apart from each other and covered with a paper bag to prevent crosscontamination. The dry rye seed were maintained at room temperature in sealed plastic bags until use.

Inoculation of plants. The nuclear tubers were washed with soap and warm water to remove excess soil deposits, then surface sterilized with $1 \% \mathrm{NaClO}$ for 10 min. The tubers were allowed to dry at room temperature and cut with a sterilized knife into 24-g pieces, on average, that included a vegetative bud. The tuber pieces were allowed to surface dry for $48 \mathrm{~h}$ at $10^{\circ} \mathrm{C}$ and to partially suberize prior to planting in order to prevent potential rot. Infested rye seed $(15 \mathrm{~g}, 650 \pm 10$ seed $)$ were added to 11 liters of potting mixture in each pot and mixed into the soil by hand to form an even distribution. Noninfested, sterile rye seed were used in the noninoculated controls. One tuber piece was planted in the middle of each pot, $1 \mathrm{~cm}$ below surface. The pots were irrigated to field capacity at planting, then were not irrigated until plant emergence. Following plant emergence, the plants were irrigated to field capacity every 2 days. The slow release fertilizer Osmocot (12 g; 16-16-16 NPK) was applied once at planting. Two months after planting (29 April 2003 and 23 April 2004), plants were pruned to one stem per plant in order to standardize the number of stems per plant among all replications. Irrigation was stopped as plants senesced and all leaves were necrotic, and the plants were allowed to die and thoroughly air dry in the pots on the greenhouse benches.

Evaluation of aggressiveness. Seven horticultural and disease severity parameters important to the Washington State potato industry were used to evaluate the aggressiveness of the isolates. The horticultural parameters were (i) total yield weight (g); (ii) number of progeny tubers; (iii) progeny tuber mean weight (g), calculated by dividing the total yield weight by the number of tubers; and (iv) specific gravity $\left(\mathrm{g} / \mathrm{cm}^{3}\right)$, calculated using the formula (total yield weight in air)/([total yield weight in air] - [total yield weight in water]).

Disease severity parameters were (i) sclerotial density on the roots and crowns $(5 \mathrm{~cm}$ of stem immediately below and above the soil level) was visually evaluated on a scale of 0 to 5 , where $0=$ no sclerotia $1=1$ to $10 \%, 2=10$ to $30 \%, 3=30$ to $50 \%, 4=50$ to $75 \%$, and $5=75$ to $100 \%$ of the surface area covered with sclerotia; (ii) height $(\mathrm{cm})$ to which sclerotia were formed on the aboveground part of the stem $(\mathrm{cm})$ was measured using a metric ruler; and (iii) incidence (\%) of infected progeny tubers, evaluated by placing vascular tissue plugs from the stolon end of the tuber onto $1 \%$ PDA and calculating the percentage of infected tubers out of 35 tubers per isolate that were sampled.

Statistical analysis. Statistical analyses were conducted only for the multimember NA-VCGs 1, 2, 3, and 5, which consisted of potato isolates. The variation in the frequencies of nitl/3 and NitM among the VCGs was analyzed as randomized complete design with VCG as the main fixed effect by contrast analysis using the logistic procedure (Proc Logistic) in SAS (SAS 9.1; SAS Institute Inc., Cary, NC). The number and frequencies of nit1/3 and NitM mutants that were generated for each VCG were calculated out of 10 or 20 repli- cations/isolate. The nonassigned isolates were not included in the analysis. The aggressiveness trials were analyzed as a randomized complete block design structure with VCGs as fixed effects, and isolates nested within VCG. The general linear models procedure (Proc GLM) in SAS (SAS 9.1; SAS Institute Inc.) was used to analyze the aggressiveness trials. Noninoculated control plants were included in the analysis of the horticultural parameters, but excluded from the analysis of the pathological parameters because they were not inoculated with the pathogen. The analysis of variance for the aggressiveness trials was followed by means separation using Fisher's protected least significant difference $t$ test. All inferences were conducted at a $5 \%$ significance level.

\section{RESULTS}

Assignment of NA isolates to VCGs. The complementation tests between the NA isolates and the EU/I VCG tester isolates were conducted in Israel during 2001-02. In all, 56 isolates from the NA collection were selected randomly and tested for vegetative compatibility with the 10 EU/I VCG testers. The complementation tests resulted in the lack of anastomosis (data not shown); therefore, we continued testing the isolates, including the 56 previously tested in Israel, at Washington State University. The NA isolates were tested for vegetative compatibility among themselves in order to select VCG testers that were specific to the NA population. Eventually, eight isolates were selected as NA-VCG testers. These isolates were selected as VCG testers based upon their ability to anastomose with multiple different isolates, and based upon their nit mutants' (nit1/nit3 and NitM) ability to react to the other isolates' nit mutants with massive prototrophic growth. The isolates that were selected as NA-VCG testers were 1018 and 1023 for NA-VCG1, 201 and 2014 for NA-VCG2, 306 for NA-VCG3, 401 for NA-VCG4, 5018 for NA-VCG5, 601 for NA-VCG6, and 701 for NAVCG7. All tester isolates demonstrated an ability to form stable heterokaryons only with isolates from their own VCG.

The complementation tests between NA isolates and the selected NA-VCG testers resulted with the assignment of 105 of 123 isolates ( $85 \%$ of the tested isolates) to seven NA-VCGs, with five multimember NA-VCGs and two two-member NAVCGs. The five multimember VCGs were NA-VCG1 with 24 isolates, NA-VCG2 with 45 isolates, NA-VCG3 with 10 isolates, NA-VCG5 with 18 isolates, and NAVCG7 with 5 isolates (Table 1). The two two-member NA-VCGs were NA-VCG4 and NA-VCG6 (Table 1). The 115 isolates originating from potato were distributed among NA-VCGs 1, 2, 3, 4 and 5 at 9, 15, 21,2 , and $38 \%$, with $17(15 \%)$ isolates not assigned to any NA-VCG (Table 1). The 
isolates originating from tomato, pepper, and mint were assigned primarily to NAVCG6 and NA-VCG7 (Table 1), excluding one mint isolate that was assigned to NAVCG2 and one mint isolate that was not assigned to any VCG (Table 1). Over all, 18 isolates (17 from potato and 1 from mint) did not react with the NA-VCG testers and were regarded as nonassigned (Table 1). We generated nit1/3 and NitM mutants to 7 of the 18 nonassigned isolates and they all were self-compatible. However, we were unable to generate NitM mutants to the remaining 11 isolates and, therefore, their self-compatibility could not be tested.

Generation and characterization of nit mutants. The nit mutants generated for the NA isolates were similar to the EU/I mutants. The mutants demonstrated thin and fast-growing mycelia on WAC media and sparse and greenish mycelia on CDA (14). The recovery time from WAC to CDA also was similar and optimal after 21 days of incubation at $25^{\circ} \mathrm{C}$ in the dark. Among the multimember NA-VCGs 1, 2, 3, and 5, which were composed primarily of potato isolates, the average frequency of nit1/nit3 class mutants was lower $(P<0.05)$ for NA-VCG1 isolates than for isolates belonging to the NA-VCGs 2, 3, and 5 (Table 2). In contrast, the frequency of NitM mutants did not vary $(P>0.05)$ among the multimember NA-VCGs $1,2,3$, and 5 and was collectively $5.14 \%$ (55 NitM of 1,070 replications) (Table 2).

The frequencies of nit1/nit3 and NitM mutants differed significantly among the EU/I-VCGs 1, 2, 3, and 4 and the NA multimember VCGs $1,2,3$, and 5 . The frequency of nitl/nit3 mutants was lower $(P<$ 0.05 ) for NA-VCGs 1,3 , and 5 than for EU/I-VCG 4. More $(P<0.05)$ nit1/nit3 mutants were generated for NA-VCG2 than for EU/I-VCG2. Fewer nit1/nit3 mutants were generated for NA-VCG1 than for EU/I-VCGs 2, 3, and 4 (Table 2). NAVCG 1 had fewer $(P<0.05)$ NitM mutants

Table 2. Frequency of nitrate-nonutilizing (nit) 1 and NitM mutants generated for European/Israeli (EU/I) and North American (NA) vegetative compatibility groups (VCGs) ${ }^{\mathrm{x}}$

\begin{tabular}{|c|c|c|c|c|}
\hline \multirow[b]{2}{*}{ VCG } & \multirow[b]{2}{*}{ No. of isolates } & \multirow[b]{2}{*}{ Replications $^{\mathbf{z}}$} & \multicolumn{2}{|c|}{ Frequency $(\%)$ of nit mutants ${ }^{y}$} \\
\hline & & & nit 1 & NitM \\
\hline EU/I-VCG1 & 8 & 80 & $33 \mathrm{BCD}$ & $9 \mathrm{AB}$ \\
\hline EU/I-VCG2 & 39 & 390 & $35 \mathrm{C}$ & $6 \mathrm{BC}$ \\
\hline EU/I-VCG3 & 22 & 220 & $44 \mathrm{AB}$ & $7 \mathrm{AB}$ \\
\hline EU/I-VCG4 & 11 & 110 & $51 \mathrm{~A}$ & $14 \mathrm{~A}$ \\
\hline NA-VCG1 & 24 & 300 & $23 \mathrm{D}$ & $3 \mathrm{C}$ \\
\hline NA-VCG2 & 45 & 500 & $42 \mathrm{AB}$ & $6 \mathrm{BC}$ \\
\hline NA-VCG3 & 10 & 100 & $36 \mathrm{BC}$ & $2 \mathrm{BC}$ \\
\hline NA-VCG5 & 17 & 170 & $39 \mathrm{BC}$ & $7 \mathrm{ABC}$ \\
\hline
\end{tabular}

${ }^{x}$ Percentage of nit 1 and NitM mutants generated for each EU/I- and NA-VCG was evaluated using a contrast analysis with the logistic procedure (Proc Logistic) in SAS at $\alpha=0.05$. The statistical model was a randomized complete design with VCG as the main fixed effect. Nonassigned isolates were not included in the analysis. Different uppercase letters within a column represent significant statistical differences among NA-VCGs.

${ }^{y}$ Frequency of nit 1 and NitM mutants generated for each VCG out of 10 to 20 replications per isolate.

${ }^{\mathrm{z}}$ Total number of replications per VCG out of 10 to 20 replications per isolate on water agar chlorate.

Table 3. Sclerotial density on root and crown, height of sclerotial formation on aboveground stem, incidence of infected progeny tubers, and total yield of Russet Norkotah potato grown in a greenhouse in 2003 and 2004 in soil infested with Colletotrichum coccodes isolates belonging to the North American vegetative compatibility groups (NA-VCGs) $1,2,3$, and $5^{\mathrm{v}}$

\begin{tabular}{lccccc}
\hline NA-VCG & $\begin{array}{c}\text { No. } \\
\text { tested }^{\mathbf{w}}\end{array}$ & $\begin{array}{c}\text { Sclerotia } \\
\text { density }^{\mathbf{x}}\end{array}$ & $\begin{array}{c}\text { Sclerotia } \\
\text { formation }(\mathbf{c m})^{\mathbf{y}}\end{array}$ & $\begin{array}{c}\text { Infected progeny } \\
\text { tubers }(\boldsymbol{\%})\end{array}$ & $\begin{array}{c}\text { Total yield } \\
\text { weight }(\mathbf{g})\end{array}$ \\
\hline 1 & 9 & $2.8 \mathrm{~b}$ & $8.4 \mathrm{c}$ & $14.7 \mathrm{~b}$ & $429 \mathrm{~b}$ \\
2 & 17 & $3.6 \mathrm{a}$ & $11.4 \mathrm{a}$ & $24.5 \mathrm{a}$ & $420 \mathrm{~b}$ \\
3 & 4 & $3.0 \mathrm{~b}$ & $6.5 \mathrm{~d}$ & $13.5 \mathrm{~b}$ & $477 \mathrm{a}$ \\
5 & 4 & $3.4 \mathrm{a}$ & $9.9 \mathrm{~b}$ & $15.0 \mathrm{~b}$ & $429 \mathrm{~b}$ \\
Control $^{\mathrm{z}}$ & $\ldots$ & 0 & 0 & 0 & $502 \mathrm{a}$ \\
\hline
\end{tabular}

${ }^{v}$ Trials were conducted in 2003 and 2004 in a randomized complete block design. The general linear model procedure (Proc GLM) in SAS was used for statistical analysis, with VCGs as the main fixed effect and isolates nested within VCGs. The analysis of variance was followed by separation of means using Fischer's protected least significant difference $t$ tests at $\alpha=0.05$. Noninoculated control plants were included only in the analysis of total yield weight. Different lowercase letters within columns represent significant statistical differences among the VCGs.

${ }^{\mathrm{w}}$ Number of isolates tested. NA-VCG1 $=9$ isolates $(38 \%$ of NA-VCG1), NA-VCG2 $=17$ isolates ( $40 \%$ of NA-VCG2), NA-VCG3 $=4$ isolates $(40 \%$ of NA-VCG3), and NA-VCG5 $=4$ isolates $(24 \%$ of NA-VCG5).

${ }^{x}$ Sclerotia density on roots and crowns was evaluated visually using the following scale: $0=$ no sclerotia, $1=1$ to $10 \%, 2=10$ to $30 \%, 3=30$ to $50 \%, 4=50$ to $75 \%$, and $5=75$ to $100 \%$ of the surface area covered with sclerotia.

${ }^{y}$ Height to which sclerotia were formed on the aboveground part of the stem.

${ }^{\mathrm{z}}$ Noninoculated control plants. than EU/I-VCGs 1,3 , and 4 and NAVCGs 2 and 3 had fewer $(P<0.05)$ NitM mutants than EU/I-VCG 4 (Table 2).

Aggressiveness trials. All of the $C$. coccodes isolates that were tested, which were distributed among the seven NAVCGs and the nonassigned and originated from potato, tomato, mint, and pepper, caused black dot on potato both years. Infection was not detected on noninoculated control plants both years. An interaction between NA-VCG and year was not recorded, and the data of the 2003 and 2004 trials was combined for the statistical analysis.

The plants infected with isolates belonging to NA-VCG2 and NA-VCG5 had more $(P<0.05)$ sclerotia on the roots and the crowns than the plants infected with isolates belonging to NA-VCGs 1 and 3 (Table 3 ). The plants infected with isolates belonging to NA-VCG2 had sclerotia that formed higher $(P<0.05)$ up the stem than the plants inoculated with isolates belonging to NA-VCGs 1,3 , or 5 (Table 3 ). The plants infected with isolates assigned to NA-VCG2 had more $(P<0.05)$ infected progeny tubers than the plants infected with isolates belonging to NA-VCGs 1,3 , or 5 (Table 3). The plants infected with isolates belonging to NA-VCGs 1, 2, and 5 had lower $(P<0.05)$ yield than the noninoculated control plants (Table 3). Significant differences among NA-VCGs were not detected for the number of progeny tubers, progeny tuber mean weight, and specific gravity. The number of progeny tubers for inoculated and noninoculated control plants ranged from 8 to 11 , tuber mean weight ranged from 40 to $74 \mathrm{~g}$, and specific gravity ranged from 1.075 to 1.088 .

A variation of aggressiveness also was recorded among isolates within VCGs. Two isolates of nine in NA-VCG1, one isolate of four in NA-VCG3, and one isolate of four in NA-VCG5 were consistently less aggressive than the other isolates (data not shown). However, the majority of the isolates with in VCGs did not differ statistically $(P>0.05)$.

\section{DISCUSSION}

The present study tested the hypothesis that the population of $C$. coccodes in North America was composed of several distinct VCGs with diverse genetic and pathological traits. The assignment of 56 NA isolates to VCGs that was conducted in Israel in 2001-02 indicated that the NA isolates did not anastomose with the VCG testers of the EU/I population (14). The results may imply that the NA and the EU/I populations were undergoing speciation, a hypothesis that should be examined and validated with a larger sample size because it may possess important disease management implications.

The assignment of NA isolates to VCGs was continued at Washington State Univer- 
sity, and multiple complementation tests among the NA isolates were conducted in order to select VCG tester isolates specific to the NA population. Eight NA isolates were selected as NA-VCG testers, and 123 NA isolates originating from potato, tomato, mint, and pepper were characterized to seven NA-VCGs distributing to five multimember groups and two two-member groups. The $115 \mathrm{NA}$ isolates originating from potato distributed among four multimember VCGs at frequencies of 9, 15, 21, and $38 \%$. Interestingly, this distribution resembled that of the EU/I isolates, which also originated from potato and distributed to four multimember VCGs at 10, 14, 28, and $49 \%$ in a similar sample size of 110 isolates (14). Furthermore, the majority of the potato isolates in both populations belonged to one large VCG (NA-VCG2 and EU/I-VCG2 in the NA and EU/I populations, respectively).

The differences recorded in relation to the frequencies of nit1/nit3 mutants indicated biological differences among North American VCGs. The results also indicated differences among NA-VCGs and EU/IVCGs, which strengthened our hypothesis that isolates from different VCGs were genetically different, and may suggest that the biochemical pathways of nitrate assimilation in $C$. coccodes were associated with subspecific groups.

As in the previous study (14) in which certain EU/I isolates did not anastomose with the VCG testers, in the present study, 18 of 123 isolates did not anastomose with the NA-VCG testers. Of the 18 isolates, 7 demonstrated self-compatibility, suggesting that the lack of interaction with the NA-VCG testers was more likely due to the presence of other VCGs that the sample size and VCG testers were unable to detect. A larger sample size and the incorporation of molecular genotyping techniques may be needed to examine this assumption.

The aggressiveness trials in the present study tested considerably more parameters than were tested previously for the EU/I population (14). The present study was the first and most comprehensive study in which $C$. coccodes isolates were tested for aggressiveness to potato based on a genetic relationship, and using yield and disease parameters of immense importance to the potato industry in Washington State. The results pointed to a variation in aggressiveness to potato which was correlated with the VCG affiliation of the NA isolates. The NA-VCGs 1, 2, and 5 demonstrated an ability to reduce yield by $16 \%$. The NAVCGs 2 and 5 demonstrated an ability to develop the most sclerotia on the roots and the crowns. On average, the isolates belonging to NA-VCG2 developed the most sclerotia up the stem, which suggested a faster colonization of the stem and, possibly, production of more sclerotia overall than the other NA-VCGs. The production of more sclerotia may point to greater fitness of NA-VCG2 isolates and may explain why it is the largest NA-VCG.

In contrast to the EU/I population in which EU/I-VCG3 (14) was clearly the most aggressive, the present study could not point to a single NA-VCG of which the isolates were, on average, the most aggressive. However, when taking into consideration all of the parameters used to assess aggressiveness, it seems that the isolates assigned to NA-VCG2 and probably those belonging to NA-VCG5 possess a greater potentially to cause disease than the isolates belonging of NA-VCGs 1 or 3 . The aggressiveness trials also indicated the ability of selected isolates from tomato, pepper, and mint to cause disease on potato. Because we tested only a limited number of these isolates, we did not compare their aggressiveness to the potato isolates from NA-VCGs 1, 2, 3, and 5. However, the results should be taken into consideration for disease management and crop rotations because, in Washington State, hosts such as mint are either rotated with potato or grown in nearby fields.

The structure of the population of $C$. coccodes and the genetic relationship among its isolates is currently under comprehensive study. In order to organize the population, we suggest the following naming system (nomenclature) based upon the continent source of the population, the VCG number, and the isolate's code. The continent prefix would be followed by the VCG number and the isolate's code. The VCG numbers would start from 1 for each continent, and the isolates' code would be arbitrary. The European/Israeli population would be designated as EU/I, and the North American population would be designated as NA. Additional subpopulations from other continents would be given the following prefixes: South America $=\mathrm{SA}$, Far East and Asia $=$ FE/A, Africa $=\mathrm{AF}$, and Australia and New Zealand $=\mathrm{A} / \mathrm{NZ}$. In this manner, isolate 24 , which is a VCG tester for VCG2 in the EU/I subpopulation (14), will be assigned as EU/I-VCG2-24; and isolate 306, which is a VCG tester for VCG3 in the NA subpopulation, will be assigned as NA-VCG3-306. We are aware of the possibility that, in the future, VCGs from different continents may be found to be similar. However, in the current stage of research, we think that this system would allow a better understanding of the relationship among the different populations.

In conclusion, the present study analyzed the population structure of the species $C$. coccodes in North America using VCGs, and the aggressiveness to potato using a large scale of genetically related isolates and numerous pathological and horticultural parameters. Seven NA-VCGs were detected among 123 C. coccodes isolates from potato, tomato, pepper, and mint, with dissimilarities in relation to genetic and aggressiveness characteristics.
Further study should quantify the relationship between the EU/I and NA populations, and examine epidemiological and etiological questions, such as host range speciation, resistance to fungicides, and effect of inoculum sources based on VCG affiliation. The results presented herein, and those previously presented $(14,15)$, documented original research that would be important to the understanding of the potato black dot pathosystem.

\section{ACKNOWLEDGMENTS}

We thank the Washington State Potato Commission for funding the present research; M. Evans, Washington State University, Department of Statistics, for his professional assistance; and N. Gudmestad from the North Dakota State University, J. Miller from the University of Idaho, S. Miller from the Ohio State University, B. Christ from The Pennsylvania State University, and T. Zitter from Cornell University for providing us with $C$. $\mathrm{coc}$ codes isolates from their collections.

\section{LITERATURE CITED}

1. Andrivon, D., Lucas, J. M., Guerin, C., and Jouan, B. 1998. Colonization of roots, stolons, tubers and stems of various potato (Solanum tuberosum) cultivars by the black dot fungus Colletotrichum coccodes. Plant Pathol. 47:440445.

2. Andrivon, D., Ramage, K., Guerin, C., Lucas, J. M., and Jouan, B. 1997. Distribution and fungicide sensitivity of Colletotrichum $\mathrm{coc}$ codes in French potato-producing areas. Plant Pathol. 46:722-728.

3. Brooker, N. L., Leslie, J. F., and Dickman, M. B. 1991. Nitrate non-utilizing mutants of $\mathrm{Col}$ letotrichum and their use in studies of vegetative compatibility and genetic relatedness. Phytopathology 81:672-677.

4. Chesters, C. G. C., and Hornby, D. 1965 Studies on Colletotrichum coccodes. I. The taxonomic significance of variation in isolates from tomato roots. Trans. Br. Mycol. Soc. 48:573-581

5. Denner, F. D. N., Millard, C., Geldenhuys, A., and Wehner, F. C. 1997. Treatment of seed potatoes with Prochloraz for simultaneous control of silver scuf and black dot on progeny tubers. Potato Res. 40:221-227.

6. Denner, F. D. N., Millard, C. P., and Wehner, F. C. 1998. The effect of seed- and soil-borne inoculum of Colletotrichum coccodes on the in cidence of black dot on potato. Potato Res. 41:51-56.

7. Dillard, H. R., and Cobb, A. C. 1998. Survival of Colletotrichum coccodes in infected tomato tissue and soil. Plant Dis. 82:235-238.

8. Joaquim, T. R., and Rowe, R. C. 1991. Vegetative compatibility and virulence of strains of Verticillium dahliae from soil and potato plants. Phytopathology 81:552-558.

9. Johnson, D. A., and Miliczky, E. R. 1993 Effect of wounding and wetting duration on infection of potato foliage by Colletotrichum coccodes. Plant Dis. 77:13-17.

10. Korolev, N., and Katan, T. 1997. Improved medium for selecting nitrate non-utilizing (nit) mutants of Verticillium dahliae. Phytopathology 87:1067-1070.

11. Korolev, N., Katan, J., and Katan, T. 2000. Vegetative compatibility groups of Verticillium dahlia in Israel: their distribution and association with pathogenicity. Phytopathology 90:529-536.

12. Lees, A. K., and Hilton, A. J. 2003. Black dot (Colletotrichum coccodes): an increasingly important disease of potato. Plant Pathol. 52:3-12.

13. Leslie, F. J. 1993. Fungal vegetative compatibility. Annu. Rev. Phytopathol. 31:127-150. 
14. Nitzan, N., Hazanovsky, M., Tal, M., and Tsror (Lahkim), L. 2002. Vegetative compatibility groups in Colletotrichum coccodes, the causal agent of black dot on potato. Phytopathology 92:827-832.

15. Nitzan, N., and Tsror (Lahkim), L. 2003. Effect of temperature and $\mathrm{pH}$ on in vitro growth rate and sclerotial density of Colletotrichum coccodes isolates from different VCGs. Am. J. Potato Res. 80:335-339.
16. Puhalla, J. E. 1979. Classification of isolates of Verticillium dahlia based on heterokaryon incompatibility. Phytopathology 69:1186-1189.

17. Puhalla, J. E., and Hummel, M. 1983. Vegetative compatibility groups within Verticillium dahlia. Phytopathology 73:1305-1308.

18. Stevenson, W. R., Green, R. J., and Bergesen, G. B. 1976. Occurrence and control of black dot root rot in Indiana. Plant Dis. Rep. 60:248251.
19. Tsror (Lahkim), L., Erlich. O., and Hazanovsky, M. 1999. Effect of Colletotrichum coccodes on potato yield, tuber quality, and stem colonization during spring and autumn. Plant Dis. 83:561-565.

20. Tsror (Lahkim), L., and Hazanovski, M. 2001 Effect of co-inoculation by Verticillium dahliae and Colletotrichum coccodes on disease symptoms and fungal colonization in four potato cultivars. Plant Pathol. 50:483-488. 